\title{
Reverse arithmetic-harmonic mean and mixed mean operator inequalities
}

Wenshi Liao* and Junliang Wu

"Correspondence:

liaowenshi@gmail.com

College of Mathematics and Statistics, Chongqing University,

Chongqing, 401331, P.R. China

\begin{abstract}
This note aims to present some scalar inequalities and operator inequalities on a Hilbert space. Firstly, the direct reverse weighted arithmetic-harmonic mean inequalities for scalars are obtained. Secondly, based on these scalar inequalities, the corresponding operator inequalities are established. Finally, we present the mixed arithmetic-geometric and geometric-harmonic means inequalities for two positive operators.
\end{abstract}

MSC: 47A63; 47A64; 47C15

Keywords: arithmetic-harmonic mean; mixed mean; Kantorovich constant; operator inequalities

\section{Introduction}

Let $\mathcal{B}(H)$ be the $C^{*}$-algebra of all bounded linear operators on a complex separable Hilbert space $H$. $I$ stands for the identity operator. $\mathcal{B}^{++}(H)$ denotes the cone of all positive invertible operators on $H$. As a matter of convenience, we use the following notations to define the $\mu$-weighted arithmetic mean (AM), geometric mean (GM), and harmonic mean (HM) for scalars and operators:

$$
\begin{aligned}
& a \nabla_{\mu} b=(1-\mu) a+\mu b, \quad a !_{\mu} b=\left((1-\mu) a^{-1}+\mu b^{-1}\right)^{-1}, \\
& A \nabla_{\mu} B=(1-\mu) A+\mu B, \quad A \#_{\mu} B=A^{\frac{1}{2}}\left(A^{-\frac{1}{2}} B A^{-\frac{1}{2}}\right)^{\mu} A^{\frac{1}{2}}, \\
& A !_{\mu} B=\left((1-\mu) A^{-1}+\mu B^{-1}\right)^{-1},
\end{aligned}
$$

where $a, b>0, A, B \in \mathcal{B}^{++}(H)$, and $\mu \in[0,1]$. When $\mu=\frac{1}{2}$, we write $a \nabla b, a ! b, A \nabla B, A \# B$ and $A ! B$ for brevity, respectively.

It is well known that the famous $\mu$-weighted A-G-H mean inequalities hold,

$$
a \nabla_{\mu} b \geq a^{1-\mu} b^{\mu} \geq a !{ }_{\mu} b
$$

for $a, b>0$ and $\mu \in[0,1]$ with equalities if and only if $a=b$. The first inequality of (1.1) is the classical Young inequality.

An operator version of (1.1) proved in [1] says that if $A, B \in \mathcal{B}^{++}(H)$ and $\mu \in[0,1]$, then

$$
A \nabla_{\mu} B \geq A \#_{\mu} B \geq A !_{\mu} B
$$

(c) 2015 Liao and Wu. This article is distributed under the terms of the Creative Commons Attribution 4.0 International License (http://creativecommons.org/licenses/by/4.0/), which permits unrestricted use, distribution, and reproduction in any medium, provided you give appropriate credit to the original author(s) and the source, provide a link to the Creative Commons license, and indicate if changes were made. 
In recent years, the study of the A-G-H mean inequalities has received increasing attention in the literature (see [2-5]).

Zuo et al. (see [5]) refined the Young inequality with the Kantorovich constant and obtained the following results:

$$
a \nabla_{\mu} b \geq \mathrm{K}(h, 2)^{r} a^{1-\mu} b^{\mu}
$$

where $a, b>0, \mu \in[0,1], r=\min \{\mu, 1-\mu\}$, and $h=\frac{b}{a}$. In addition, they also refined the $\mu$-weighted arithmetic-harmonic mean inequality and extended it to an operator version as follows:

$$
\begin{aligned}
& a \nabla_{\mu} b \geq a !_{\mu} b+2 r(a \nabla b-a ! b), \\
& A \nabla_{\mu} B \geq A !_{\mu} B+2 r(A \nabla B-A ! B),
\end{aligned}
$$

where $a, b>0, A, B \in \mathcal{B}^{++}(H), \mu \in[0,1]$, and $r=\min \{\mu, 1-\mu\}$. By (1.3) and (1.4), we are encouraged to investigate whether there exist reverse forms of the $\mu$-weighted arithmeticharmonic mean inequality, so we give an affirmative answer to this question in our paper.

Moreover, mixed mean inequalities are also extremely attractive. Sagae and Tanabe [6] establish a mixed A-G mean inequality for a finite number of strictly positive operators. Mond and Pečarić [7] establish a mixed A-G and G-H mean inequalities for two noncommutative strictly positive operators as follows: Let $\mathrm{A}$ and $\mathrm{B}$ be positive invertible operators. The mixed arithmetic-geometric and geometric-harmonic mean inequalities are valid:

$$
\begin{aligned}
& A \#(A \nabla B) \geq A \nabla(A \# B), \\
& A \#(A ! B) \leq A !(A \# B) .
\end{aligned}
$$

In this paper, we are concerned with the weighted arithmetic-harmonic, mixed arithmetic-geometric and mixed geometric-harmonic mean inequalities. In Section 2, we present direct reverse weighted arithmetic-harmonic mean inequalities by the Kantorovich constant and deduce some auxiliary results. In Section 3, we extend inequalities proved in Section 2 from the scalars setting to a Hilbert space operator setting. In Section 4, we establish mixed weighted arithmetic-geometric and geometric-harmonic means inequalities for two positive operators which are the refinements of (1.5) and (1.6).

\section{Reverse arithmetic-harmonic mean inequalities}

In this section, we mainly present the direct reverse forms of the $\mu$-weighted arithmeticharmonic mean inequality for two positive numbers $a, b$.

First of all, we recall the classical Kantorovich inequality in [8].

Lemma 2.1 Let $0<a=x_{1}<x_{2}<\cdots<x_{n}=b$ be given positive numbers, $\lambda_{1}, \lambda_{2}, \ldots, \lambda_{n} \geq 0$ and $\sum_{i=1}^{n} \lambda_{i}=1$. Then

$$
\left(\sum_{i=1}^{n} \lambda_{i} x_{i}\right)\left(\sum_{i=1}^{n} \lambda_{i} x_{i}^{-1}\right) \leq A^{2} G^{-2},
$$

where $A=\frac{1}{2}(a+b), G=\sqrt{a b}$ are the arithmetic and geometric means, respectively. 
The inequality (2.1) is the Kantorovich inequality and the number $\frac{(a+b)^{2}}{4 a b}$ is called the Kantorovich constant. For convenience, we write the Kantorovich constant as $\mathrm{K}(t, 2)=$ $\frac{(t+1)^{2}}{4 t}\left(t=\frac{b}{a}\right)$, which has the properties $\mathrm{K}(1,2)=1, \mathrm{~K}(t, 2)=\mathrm{K}\left(\frac{1}{t}, 2\right) \geq 1(t>0)$, and $\mathrm{K}(t, 2)$ is monotone increasing on $[1, \infty)$, and monotone decreasing on $(0,1]$.

When $n=2$, we can get a special form for the inequality (2.1) as follows.

Proposition 2.1 Let $a, b>0$ and $\mu \in[0,1]$. Then

$$
a \nabla_{\mu} b \leq \mathrm{K}(h, 2) a{ }_{\mu} b
$$

where $h=\frac{b}{a}$. Equality holds if and only if $a=b$.

Remark 2.1 The inequality (2.2) is a direct reverse of the $\mu$-weighted arithmeticharmonic mean inequality. It is very interesting that by the inequality (2.2) and (1.2) we can get a reverse of Young inequality (it is also a reverse of the inequality (1.2)):

$$
a \nabla_{\mu} b \leq \mathrm{K}(h, 2) a !_{\mu} b \leq \mathrm{K}(h, 2)^{1-r} a^{1-\mu} b^{\mu}=\mathrm{K}(h, 2)^{R} a^{1-\mu} b^{\mu},
$$

where $a, b>0, \mu \in[0,1], r=\min \{\mu, 1-\mu\}$, and $R=\max \{1-\mu, \mu\}$. Replacing $a, b$ by $a^{-1}$, $b^{-1}$ in the above inequalities, respectively, we have

$$
a^{1-\mu} b^{\mu} \leq \mathrm{K}(h, 2)^{R} a !_{\mu} b .
$$

Next, we deduce a direct reverse of the inequality (1.3) by the following lemma.

Lemma 2.2 [9] Let $x_{i}(i=1,2, \ldots, n)$ belong to a fixed closed interval $\mathbb{I}, p_{i} \geq 0$ with $\sum_{i=1}^{n} p_{i}=$ 1 and $\bar{p}=\max \left\{p_{1}, p_{2}, \ldots, p_{n}\right\}$. Iff is a convex function on $\mathbb{I}$, then

$$
\sum_{i=1}^{n} p_{i} f\left(x_{i}\right)-f\left(\sum_{i=1}^{n} p_{i} x_{i}\right) \leq n \bar{p}\left[\sum_{i=1}^{n} \frac{1}{n} f\left(x_{i}\right)-f\left(\sum_{i=1}^{n} \frac{1}{n} x_{i}\right)\right] .
$$

It is easy to see that if we take $f(x)=x^{-1}$ in the inequality (2.5), then we have the following.

Proposition 2.2 If $x_{1}, x_{2}, \ldots, x_{n}>0$ and $p_{i} \geq 0(i=1,2, \ldots, n)$ with $\sum_{i=1}^{n} p_{i}=1$, then

$$
\sum_{i=1}^{n} p_{i} x_{i}^{-1}-\left(\sum_{i=1}^{n} p_{i} x_{i}\right)^{-1} \leq n \bar{p}\left[\sum_{i=1}^{n} \frac{1}{n} x_{i}^{-1}-\left(\sum_{i=1}^{n} \frac{1}{n} x_{i}\right)^{-1}\right],
$$

where $\bar{p}=\max \left\{p_{1}, p_{2}, \ldots, p_{n}\right\}$.

In particular, when $n=2$ in the inequality (2.6), we can get

$$
a \nabla_{\mu} b \leq a !_{\mu} b+2 R(a \nabla b-a ! b)
$$

where $a, b>0, \mu \in[0,1], R=\max \{1-\mu, \mu\}$. Equality holds if and only if $a=b$.

Note that (2.7) is a reverse of the inequality (1.3) with a similar form.

The following three theorems are our main reverse forms of the $\mu$-weighted arithmeticharmonic mean inequality for scalars. 
Theorem 2.1 Let $a, b>0$, and $\mu \in[0,1]$. Then the inequality

$$
a \nabla_{\mu} b \leq 2 r(a \nabla b-a ! b)+\mathrm{K}(\sqrt{h}, 2)^{R^{\prime}} \mathrm{K}(h, 2)^{R} a !_{\mu} b
$$

holds, where $h=\frac{b}{a}, r=\min \{\mu, 1-\mu\}, R=\max \{\mu, 1-\mu\}$, and $R^{\prime}=\max \{2 r, 1-2 r\}$. Equality holds if and only if $a=b$.

Proof By the inequalities (2.3) and (2.4), firstly, we consider the case $\mu \in\left[0, \frac{1}{2}\right]$,

$$
\begin{aligned}
a \nabla_{\mu} b-2 \mu(a \nabla b-a ! b) & \leq a \nabla_{\mu} b-2 \mu(a \nabla b-\sqrt{a b}) \\
& =(1-2 \mu) a+2 \mu \sqrt{a b} \\
& \leq \mathrm{K}(\sqrt{h}, 2)^{R^{\prime}} a^{1-\mu} b^{\mu} \\
& \leq \mathrm{K}(\sqrt{h}, 2)^{R^{\prime}} \mathrm{K}(h, 2)^{R} a !_{\mu} b .
\end{aligned}
$$

If $\mu \in\left(\frac{1}{2}, 1\right]$, then we have

$$
\begin{aligned}
a \nabla_{\mu} b-2(1-\mu)(a \nabla b-a ! b) & \leq a \nabla_{\mu} b-2(1-\mu)(a \nabla b-\sqrt{a b}) \\
& =(2 \mu-1) b+2(1-\mu) \sqrt{a b} \\
& \leq \mathrm{K}(\sqrt{h}, 2)^{R^{\prime}} a^{1-\mu} b^{\mu} \\
& \leq \mathrm{K}(\sqrt{h}, 2)^{R^{\prime}} \mathrm{K}(h, 2)^{R} a ! \mu b .
\end{aligned}
$$

By the above discussion, for any $\mu \in[0,1]$, the inequality (2.8) always holds.

Note that (2.8) can also be considered as a reverse ratio inequality of (1.3):

$$
0<a !_{\mu} b \leq a \nabla_{\mu} b-2 r(a \nabla b-a ! b) \leq \mathrm{K}(\sqrt{h}, 2)^{R^{\prime}} \mathrm{K}(h, 2)^{R} a !_{\mu} b .
$$

Theorem 2.2 Let $a, b>0, \mu \in[0,1]$, and $h=\frac{b}{a}$.

(I) If $0 \leq \mu \leq \frac{1}{2}$, then

$$
a \nabla_{\mu} b-2 \mu(a \nabla b-a ! b) \leq \mathrm{K}(\sqrt{h}, 2) a !_{2 \mu} \sqrt{a b} .
$$

Equality holds if and only if $a=b$.

(II) If $\frac{1}{2}<\mu \leq 1$, then

$$
a \nabla_{\mu} b-2(1-\mu)(a \nabla b-a ! b) \leq \mathrm{K}(\sqrt{h}, 2) b !_{2-2 \mu} \sqrt{a b} .
$$

Equality holds if and only if $a=b$.

Proof By the inequality (2.2), firstly, we consider the case $\mu \in\left[0, \frac{1}{2}\right]$, then we have

$$
\begin{aligned}
a \nabla_{\mu} b-2 \mu(a \nabla b-a ! b) & \leq a \nabla_{\mu} b-2 \mu(a \nabla b-\sqrt{a b}) \\
& =(1-2 \mu) a+2 \mu \sqrt{a b} \\
& \leq \mathrm{K}(\sqrt{h}, 2) a !_{2 \mu} \sqrt{a b} .
\end{aligned}
$$


If $\mu \in\left(\frac{1}{2}, 1\right]$, then we have

$$
\begin{aligned}
a \nabla_{\mu} b-2(1-\mu)(a \nabla b-a ! b) & \leq a \nabla_{\mu} b-2(1-\mu)(a \nabla b-\sqrt{a b}) \\
& =(2 \mu-1) b+2(1-\mu) \sqrt{a b} \\
& \leq \mathrm{K}(\sqrt{h}, 2) b !_{2-2 \mu} \sqrt{a b}
\end{aligned}
$$

The proof is completed.

Theorem 2.3 Let $a, b>0$ and $\mu \in[0,1]$.

(I) If $0 \leq \mu \leq \frac{1}{2}$, then

$$
a \nabla_{\mu} b-2 \mu(a \nabla b-a ! b) \leq a !_{2 \mu} \sqrt{a b}+2 R^{\prime}(a \nabla \sqrt{a b}-a ! \sqrt{a b}) .
$$

Equality holds if and only if $a=b$.

(II) If $\frac{1}{2}<\mu \leq 1$, then

$$
a \nabla_{\mu} b-2(1-\mu)(a \nabla b-a ! b) \leq b !_{2-2 \mu} \sqrt{a b}+2 R^{\prime}(b \nabla \sqrt{a b}-b ! \sqrt{a b}) .
$$

Equality holds if and only if $a=b$.

Proof Firstly, we consider the case $\mu \in\left[0, \frac{1}{2}\right]$, by the inequality (2.7), then we have

$$
\begin{aligned}
a \nabla_{\mu} b-2 \mu(a \nabla b-a ! b) & \leq(1-2 \mu) a+2 \mu \sqrt{a b} \\
& \leq a !_{2 \mu} \sqrt{a b}+2 \max \{1-2 \mu, 2 \mu\}(a \nabla \sqrt{a b}-a ! \sqrt{a b}) .
\end{aligned}
$$

If $\mu \in\left(\frac{1}{2}, 1\right]$, by the inequality (2.7), then we have

$$
\begin{aligned}
& a \nabla_{\mu} b-2(1-\mu)(a \nabla b-a ! b) \\
& \quad \leq(2 \mu-1) b+2(1-\mu) \sqrt{a b} \\
& \quad \leq b !_{2-2 \mu} \sqrt{a b}+2 \max \{2-2 \mu, 2 \mu-1\}(b \nabla \sqrt{a b}-b ! \sqrt{a b}) .
\end{aligned}
$$

The proof is completed.

Note that the inequalities proved in Theorem 2.1-2.3 are all the reverses of the inequality (1.3).

\section{Reverse arithmetic-harmonic mean operator inequalities}

In this section, we present the operator versions of these reverse arithmetic-harmonic mean inequalities proved in Section 2. The techniques are based on the monotonicity property of operator functions described in the following lemma (for more details, see $[1,10])$.

Lemma 3.1 Let $X \in \mathcal{B}(H)$ be self-adjoint operator and if $f$ and $g$ are both continuous functions with $f(t) \geq g(t)$ for $t \in \operatorname{Sp}(X)$ (the spectrum of $X$ ), then $f(X) \geq g(X)$ with equality if and only if $f(t)=g(t)$ for all $t \in \operatorname{Sp}(X)$. 
Based on Proposition 2.1, we can deduce the operator inequality which is also the noncommutative Kantorovich inequality proved by Furuta et al. [1], but our method is different.

Proposition 3.1 Let $A, B \in \mathcal{B}^{++}(H)$ and positive real numbers $m$, $M$ satisfy $0<m I \leq A, B \leq$ MI. Then

$$
A \nabla_{\mu} B \leq \mathrm{K}(h, 2) A !_{\mu} B
$$

where $\mu \in[0,1]$ and $h=\frac{M}{m}$. Equality holds if and only if $A=B$ and $m=M$.

Proof By the inequality (2.2), we have

$$
(1-\mu)+\mu x \leq \mathrm{K}(x, 2)\left((1-\mu)+\mu x^{-1}\right)^{-1}
$$

for any $x>0$, and hence

$$
(1-\mu) I+\mu X \leq \max _{\frac{1}{h} \leq x \leq h} \mathrm{~K}(x, 2)\left((1-\mu) I+\mu X^{-1}\right)^{-1}
$$

for the positive operator $X$ such that $0<\frac{1}{h} I \leq X \leq h I$.

Since $0<\frac{1}{h} I \leq A^{-\frac{1}{2}} B A^{-\frac{1}{2}} \leq h I$ and the Kantorovich constant $K(t, 2)$ is an increasing function for $t>1$ and $\mathrm{K}\left(\frac{1}{h}, 2\right)=\mathrm{K}(h, 2)$, substituting $A^{-\frac{1}{2}} B A^{-\frac{1}{2}}$ for $X$ in the above inequality, we have

$$
(1-\mu) I+\mu A^{-\frac{1}{2}} B A^{-\frac{1}{2}} \leq \mathrm{K}(h, 2)\left((1-\mu) I+\mu\left(A^{-\frac{1}{2}} B A^{-\frac{1}{2}}\right)^{-1}\right)^{-1} .
$$

Multiplying both sides of the above inequality by $A^{\frac{1}{2}}$, we can deduce the required inequality (3.1).

We prove an operator inequality obtained by Krnić et al. in [11] by a different method. But our method is more transparent and simpler than the one given in [11].

Proposition 3.2 Let $A, B \in \mathcal{B}^{++}(H)$ and $\mu \in[0,1]$. Then

$$
A \nabla_{\mu} B \leq A !_{\mu} B+2 R(A \nabla B-A ! B),
$$

where $R=\max \{1-\mu, \mu\}$. Equality holds if and only if $A=B$.

Proof By the inequality (2.7), for $x>0$ and $\mu \in[0,1]$, we have

$$
(1-\mu)+\mu x^{-1} \leq((1-\mu)+\mu x)^{-1}+2 R\left[\frac{1+x^{-1}}{2}-\left(\frac{1+x}{2}\right)^{-1}\right]
$$

For a positive invertible operator $T$ and $\mu \in[0,1]$, it follows that

$$
(1-\mu) I+\mu T^{-1} \leq((1-\mu) I+\mu T)^{-1}+2 R\left[\frac{I+T^{-1}}{2}-\left(\frac{I+T}{2}\right)^{-1}\right]
$$


Putting $T=A^{-\frac{1}{2}} B A^{-\frac{1}{2}}$ in the above inequality and multiplying both sides by $A^{\frac{1}{2}}$, we deduce the inequality (3.2).

Note that, by (1.4) and (3.2), we have

$$
\begin{aligned}
0 & <A !_{\mu} B \\
& \leq A !_{\mu} B+2 r(A \nabla B-A ! B) \\
& \leq A \nabla_{\mu} B \\
& \leq A !_{\mu} B+2 R(A \nabla B-A ! B) .
\end{aligned}
$$

Based on Theorem 2.1, we have the following.

Theorem 3.1 Let $A, B \in \mathcal{B}^{++}(H)$ and positive real numbers $m, M$ satisfy $0<m I \leq A, B \leq$ $M I$. Then for $\mu \in[0,1]$,

$$
A \nabla_{\mu} B-2 r(A \nabla B-A ! B) \leq \mathrm{K}(\sqrt{h}, 2)^{R^{\prime}} \mathrm{K}(h, 2)^{R} A !_{\mu} B
$$

where $h=\frac{M}{m}, r=\min \{\mu, 1-\mu\}, R=\max \{\mu, 1-\mu\}$, and $R^{\prime}=\max \{2 r, 1-2 r\}$. Equality holds if and only if $A=B$ and $m=M$.

Proof By the inequality (2.8), we have

$$
\begin{gathered}
(1-\mu)+\mu x-2 r\left[\frac{1+x}{2}-\left(\frac{1}{2}+\frac{1}{2} x^{-1}\right)^{-1}\right] \\
\leq \mathrm{K}(\sqrt{x}, 2)^{R^{\prime}} \mathrm{K}(x, 2)^{R}\left((1-\mu)+\mu x^{-1}\right)^{-1}
\end{gathered}
$$

for any $x>0$, and hence

$$
\begin{gathered}
(1-\mu) I+\mu X-2 r\left[\frac{I+X}{2}-\left(\frac{1}{2} I+\frac{1}{2} X^{-1}\right)^{-1}\right] \\
\leq \mathrm{K}(\sqrt{h}, 2)^{R^{\prime}} \mathrm{K}(h, 2)^{R}\left((1-\mu) I+\mu X^{-1}\right)^{-1}
\end{gathered}
$$

for the positive operator $X$ such that $0<\frac{1}{h} I \leq X \leq h I$.

By a similar process to Proposition 3.1, we can deduce the required inequality (3.3).

Note that (3.3) is a reverse of (1.4):

$$
0<A !_{\mu} B \leq A \nabla_{\mu} B-2 r(A \nabla B-A ! B) \leq \mathrm{K}(\sqrt{h}, 2)^{R^{\prime}} \mathrm{K}(h, 2)^{R} A !_{\mu} B .
$$

Now, we exhibit the operator inequalities based on Theorem 2.2 and Theorem 2.3.

Theorem 3.2 Let $A, B \in \mathcal{B}^{++}(H)$ and $\mu \in[0,1]$. The positive real numbers $m$, $M$ satisfy $0<m I \leq A, B \leq M I$ and $h=\frac{M}{m}$. 
(I) If $0 \leq \mu \leq \frac{1}{2}$, then

$$
A \nabla_{\mu} B-2 \mu(A \nabla B-A ! B) \leq \mathrm{K}(\sqrt{h}, 2) A !_{2 \mu}(A \# B)
$$

Equality holds if and only if $A=B$ and $m=M$.

(II) If $\frac{1}{2}<\mu \leq 1$, then

$$
A \nabla_{\mu} B-2(1-\mu)(A \nabla B-A ! B) \leq \mathrm{K}(\sqrt{h}, 2) B !_{2(1-\mu)}(A \# B) .
$$

Equality holds if and only if $A=B$ and $m=M$.

Proof If $0 \leq \mu \leq \frac{1}{2}$, by (I) of Theorem 2.2, we have

$$
\begin{gathered}
(1-\mu)+\mu a-2 \mu\left[\frac{1+a}{2}-\left(\frac{1}{2}+\frac{1}{2} a^{-1}\right)^{-1}\right] \\
\leq \mathrm{K}(\sqrt{a}, 2)\left[(1-2 \mu)+2 \mu a^{-\frac{1}{2}}\right]^{-1}
\end{gathered}
$$

for any $a>0$, and hence

$$
\begin{gathered}
(1-\mu) I+\mu X-2 \mu\left[\frac{I+X}{2}-\left(\frac{1}{2} I+\frac{1}{2} X^{-1}\right)^{-1}\right] \\
\leq \mathrm{K}(\sqrt{h}, 2)\left[(1-2 \mu) I+2 \mu X^{-\frac{1}{2}}\right]^{-1}
\end{gathered}
$$

for the positive invertible operator $X$ such that $0<\frac{1}{h} I \leq X \leq h I$.

Substituting $A^{-\frac{1}{2}} B A^{-\frac{1}{2}}$ for $X$ in the above inequality, we have

$$
\begin{aligned}
& (1-\mu) I+\mu A^{-\frac{1}{2}} B A^{-\frac{1}{2}} \\
& \quad-2 \mu\left[\frac{I+A^{-\frac{1}{2}} B A^{-\frac{1}{2}}}{2}-\left(\frac{1}{2} I+\frac{1}{2}\left(A^{-\frac{1}{2}} B A^{-\frac{1}{2}}\right)^{-1}\right)^{-1}\right] \\
& \leq \mathrm{K}(\sqrt{h}, 2)\left[(1-2 \mu) I+2 \mu\left(A^{-\frac{1}{2}} B A^{-\frac{1}{2}}\right)^{-\frac{1}{2}}\right]^{-1} .
\end{aligned}
$$

Multiplying both sides of (3.6) by $A^{\frac{1}{2}}$, we can deduce the required inequality (3.4).

Likewise, if $\frac{1}{2}<\mu \leq 1$, by (II) of Theorem 2.2 , we have

$$
\begin{gathered}
(1-\mu) b+\mu-2(1-\mu)\left[\frac{1+b}{2}-\left(\frac{1}{2}+\frac{1}{2} b^{-1}\right)^{-1}\right] \\
\leq \mathrm{K}(\sqrt{b}, 2)\left[(2 \mu-1) b^{-\frac{1}{2}}+(2-2 \mu)\right]^{-1}
\end{gathered}
$$

for any $b>0$, and hence

$$
\begin{gathered}
(1-\mu) Y+\mu I-2(1-\mu)\left[\frac{I+Y}{2}-\left(\frac{1}{2} I+\frac{1}{2} Y^{-1}\right)^{-1}\right] \\
\leq \mathrm{K}(\sqrt{h}, 2)\left[(2 \mu-1) Y^{-\frac{1}{2}}+(2-2 \mu) I\right]^{-1}
\end{gathered}
$$

for the positive invertible operator $Y$ such that $0<\frac{1}{h} I \leq Y \leq h I$. 
Substituting $B^{-\frac{1}{2}} A B^{-\frac{1}{2}}$ for $Y$ in the above inequality, we have

$$
\begin{aligned}
\mu I+ & (1-\mu) B^{-\frac{1}{2}} A B^{-\frac{1}{2}} \\
& -2(1-\mu)\left[\frac{I+B^{-\frac{1}{2}} A B^{-\frac{1}{2}}}{2}-\left(\frac{1}{2} I+\frac{1}{2} I\left(B^{-\frac{1}{2}} A B^{-\frac{1}{2}}\right)^{-1}\right)^{-1}\right] \\
\leq & K(\sqrt{h}, 2)\left[(2 \mu-1)\left(B^{-\frac{1}{2}} A B^{-\frac{1}{2}}\right)^{-\frac{1}{2}}+(2-2 \mu) I\right]^{-1} .
\end{aligned}
$$

Multiplying both sides of (3.7) by $B^{\frac{1}{2}}$, we can deduce the required inequality (3.5).

Note that, by (1.4), (3.4), and (3.5), we have

$$
\begin{aligned}
0 & <A !_{\mu} B \\
& \leq A \nabla_{\mu} B-2 r(A \nabla B-A ! B) \\
& \leq \begin{cases}K(\sqrt{h}, 2) A !_{2 \mu}(A \# B), & 0 \leq \mu \leq \frac{1}{2}, \\
K(\sqrt{h}, 2) B !_{2(1-\mu)}(A \# B), & \frac{1}{2}<\mu \leq 1 .\end{cases}
\end{aligned}
$$

Theorem 3.3 Let $A, B \in \mathcal{B}^{++}(H)$ and $\mu \in[0,1]$.

(I) If $0 \leq \mu \leq \frac{1}{2}$, then

$$
A \nabla_{\mu} B-2 \mu(A \nabla B-A ! B) \leq A !_{2 \mu}(A \# B)+2 R^{\prime}(A \nabla(A \# B)-A !(A \# B)) .
$$

Equality holds if and only if $A=B$.

(II) If $\frac{1}{2}<\mu \leq 1$, then

$$
A \nabla_{\mu} B-2(1-\mu)(A \nabla B-A ! B) \leq B !_{2-2 \mu}(A \# B)+2 R^{\prime}(B \nabla(A \# B)-B !(A \# B)) .
$$

Equality holds if and only if $A=B$.

Proof If $0 \leq \mu \leq \frac{1}{2}$, by (I) of Theorem 2.3, we have

$$
\begin{aligned}
& (1-\mu)+\mu b-2 \mu\left[\frac{1+b}{2}-\left(\frac{1}{2}+\frac{1}{2} b^{-1}\right)^{-1}\right]-\left[(1-2 \mu)+2 \mu b^{-\frac{1}{2}}\right]^{-1} \\
& \quad \leq 2 \max \{1-2 \mu, 2 \mu\}\left[\frac{1}{2}+\frac{1}{2} b^{\frac{1}{2}}-\left(\frac{1}{2}+\frac{1}{2} b^{-\frac{1}{2}}\right)^{-1}\right]
\end{aligned}
$$

for any $b>0$, and hence

$$
\begin{aligned}
& (1-\mu) I+\mu X-2 \mu\left[\frac{I+X}{2}-\left(\frac{1}{2} I+\frac{1}{2} X^{-1}\right)^{-1}\right]-\left[(1-2 \mu) I+2 \mu X^{-\frac{1}{2}}\right]^{-1} \\
& \quad \leq 2 \max \{1-2 \mu, 2 \mu\}\left[\frac{1}{2} I+\frac{1}{2} X^{\frac{1}{2}}-\left(\frac{1}{2} I+\frac{1}{2} X^{-\frac{1}{2}}\right)^{-1}\right]
\end{aligned}
$$

for the positive invertible operator $X$.

Substituting $A^{-\frac{1}{2}} B A^{-\frac{1}{2}}$ for $X$ in the above inequality and then multiplying both sides by $A^{\frac{1}{2}}$, we can deduce the required inequality (3.8). 
Likewise, if $\frac{1}{2}<\mu \leq 1$, by (II) of Theorem 2.3, we have

$$
\begin{aligned}
& (1-\mu) a+\mu-2(1-\mu)\left[\frac{a+1}{2}-\left(\frac{1}{2} a^{-1}+\frac{1}{2}\right)^{-1}\right] \\
& \quad \leq\left[(2 \mu-1)+(2-2 \mu) a^{-\frac{1}{2}}\right]^{-1}+2 \max \{2-2 \mu, 2 \mu-1\}\left[\frac{1}{2}+\frac{1}{2} a^{\frac{1}{2}}-\left(\frac{1}{2}+\frac{1}{2} a^{-\frac{1}{2}}\right)^{-1}\right]
\end{aligned}
$$

for any $a>0$, and hence

$$
\begin{aligned}
(1-\mu) Y+\mu I-2(1-\mu)\left[\frac{Y+I}{2}-\left(\frac{1}{2} Y^{-1}+\frac{1}{2} I\right)^{-1}\right] \\
\leq \\
\quad\left[(2 \mu-1) I+(2-2 \mu) Y^{-\frac{1}{2}}\right]^{-1} \\
\quad+2 \max \{2-2 \mu, 2 \mu-1\}\left[\frac{1}{2} I+\frac{1}{2} Y^{\frac{1}{2}}-\left(\frac{1}{2} I+\frac{1}{2} Y^{-\frac{1}{2}}\right)^{-1}\right]
\end{aligned}
$$

for the positive invertible operator $Y$.

Substituting $B^{-\frac{1}{2}} A B^{-\frac{1}{2}}$ for $Y$ in the above inequality and then multiplying both sides by $B^{\frac{1}{2}}$, we can deduce the required inequality (3.9).

Note that, by (1.4), (3.8), and (3.9), we have

$$
\begin{aligned}
0 & <A !_{\mu} B \\
& \leq A \nabla_{\mu} B-2 r(A \nabla B-A ! B) \\
& \leq \begin{cases}A !_{2 \mu}(A \# B)+2 R^{\prime}(A \nabla(A \# B)-A !(A \# B)), & 0 \leq \mu \leq \frac{1}{2}, \\
B !_{2-2 \mu}(A \# B)+2 R^{\prime}(B \nabla(A \# B)-B !(A \# B)), & \frac{1}{2}<\mu \leq 1 .\end{cases}
\end{aligned}
$$

Remark 3.1 These inequalities proved in Theorem 3.1-3.3 are all the reverse forms of the inequality (1.4). It is easy to see that the right-hand side of these inequalities can not be compared with each other, but they are indeed new versions of reverse ratio arithmeticharmonic mean inequality.

\section{The mixed mean inequalities}

In this section, we obtain refinements of the inequalities (1.5) and (1.6) and deduce some mixed weighted arithmetic-geometric and geometric-harmonic means inequalities.

First, we need the following lemma.

Lemma 4.1 (Hermite-Hadamard's inequality [12]) Iff $: \mathbb{I} \rightarrow \mathbb{R}$ is a convex function on the interval $\mathbb{I} \subset \mathbb{R}$, then for any $x, y \in \mathbb{I}$ with $x \neq y$, we have

$$
f\left(\frac{x+y}{2}\right) \leq \frac{1}{y-x} \int_{x}^{y} f(t) d t \leq \frac{f(x)+f(y)}{2} .
$$

Theorem 4.1 Let $A, B \in \mathcal{B}^{++}(H)$. Then

$$
\begin{aligned}
& A \#(A \nabla B) \geq \frac{1}{3}\left[4 A \nabla(A \# B)-(A \# B)(A \nabla(A \# B))^{-1} A\right] \geq A \nabla(A \# B), \\
& A \#(A ! B) \leq 3\left[4(A !(A \# B))^{-1}-(A \# B)^{-1}(A !(A \# B)) A^{-1}\right]^{-1} \leq A !(A \# B) .
\end{aligned}
$$


Proof Applying the Hermite-Hadamard's inequalities (4.1) to the convex function $f(t)=$ $-t^{\frac{1}{2}}, t>0$, we have

$$
\left(\frac{x+y}{2}\right)^{1 / 2} \geq \frac{1}{3}\left[4\left(\frac{x^{1 / 2}+y^{1 / 2}}{2}\right)-(x y)^{1 / 2}\left(\frac{x^{1 / 2}+y^{1 / 2}}{2}\right)^{-1}\right] \geq \frac{\left(x^{1 / 2}+y^{1 / 2}\right)}{2}
$$

and hence

$$
\left(\frac{I+C}{2}\right)^{1 / 2} \geq \frac{1}{3}\left[4\left(\frac{I+C^{1 / 2}}{2}\right)-(C)^{1 / 2}\left(\frac{I+C^{1 / 2}}{2}\right)^{-1}\right] \geq \frac{\left(I+C^{1 / 2}\right)}{2}
$$

for a positive invertible operator $C$.

Putting $C=A^{-\frac{1}{2}} B A^{-\frac{1}{2}}$ in the above inequality and multiplying both sides by $A^{\frac{1}{2}}$, we can obtain (4.2).

Substituting $A^{-1}$ for $A$ and $B^{-1}$ for $B$ and then taking the inverse in (4.2), we get (4.3).

Note that the inequalities (4.2) and (4.3) are the refinements of (1.5) and (1.6), respectively.

If $f$ is convex on a segment $[a, b]$ of a linear space, one can easily observe that (4.1) is equivalent to the following double inequality:

$$
f\left(\frac{a+b}{2}\right) \leq \int_{0}^{1} f((1-t) a+t b) d t \leq \frac{f(a)+f(b)}{2} .
$$

A natural generalization of the classical Hermite-Hadamard inequality to Hermitian matrices could be the double inequality

$$
f\left(\frac{A+B}{2}\right) \leq \int_{0}^{1} f((1-t) A+t B) d t \leq \frac{f(A)+f(B)}{2} .
$$

However, Moslehian [13] pointed out that this was not true. We will show that the following result is valid.

Theorem 4.2 Let $A, B \in \mathcal{B}^{++}(H)$. Then

$$
A \#(A \nabla B) \geq \int_{0}^{1} A \#\left(A \nabla_{t} B\right) d t \geq A \nabla(A \# B)
$$

Proof Taking $f(t)=-t^{\frac{1}{2}}, t>0$ in (4.4), then

$$
\left(\frac{a+b}{2}\right)^{1 / 2} \geq \int_{0}^{1}((1-t) a+t b)^{1 / 2} d t \geq \frac{\left(a^{1 / 2}+b^{1 / 2}\right)}{2}
$$

and by a method resembling Theorem 4.1, we obtain (4.5).

In the next theorems, mixed weighted arithmetic-geometric and geometric-harmonic mean inequalities are established. First, we show the definition of operator convex (see [10], p.113). 
Definition 4.1 A continuous function $f: \mathbb{I} \rightarrow \mathbb{R}$ on the interval $\mathbb{I} \subset \mathbb{R}$ is said to be operator convex if for every pair of self-adjoint operators $X, Y$ on a Hilbert space $H$ with spectrum in $\mathbb{I}$ and each $v \in[0,1]$,

$$
f((1-v) X+v Y) \leq(1-v) f(X)+v f(Y)
$$

A function $f$ is called operator concave if the function $-f$ is operator convex. The function $f(X)=X^{s}$ is operator convex on a self-adjoint operator space for $-1 \leq s \leq 0$ or $1 \leq s \leq 2$ and is operator concave for $0 \leq s \leq 1$.

For an operator convex function $f: \mathbb{I} \rightarrow \mathbb{R}$ on the interval $\mathbb{I} \subset \mathbb{R}$, we have the following property (see [14], p.71): For each $v \notin[0,1]$, self-adjoint operators $X, Y$ and $(1-v) X+v Y$ with spectra in $\mathbb{I}$,

$$
f((1-v) X+v Y) \geq(1-v) f(X)+v f(Y)
$$

In the next theorems, we still use the notations $A \nabla_{v} B, v \notin[0,1]$, and $A \#_{s} B, s \in[-1,0] \cup$ $[1,2]$.

Theorem 4.3 Let $A, B \in \mathcal{B}^{++}(H)$, and $v \in[0,1]$. If $s \in[-1,0] \cup[1,2]$, then

$$
\begin{aligned}
& A \#_{s}\left(A \nabla_{v} B\right) \leq A \nabla_{v}\left(A \#_{s} B\right), \\
& A \#_{S}\left(A !_{v} B\right) \geq A !_{v}\left(A \#_{s} B\right) .
\end{aligned}
$$

Proof By the inequality (4.6), for every pair of self-adjoint operators $X, Y$ and the operator convex function $f(x)=x^{s}(x>0), s \in[-1,0] \cup[1,2]$, we have

$$
[(1-v) X+v Y]^{s} \leq(1-v) X^{s}+v Y^{s}
$$

Putting $X=I$ and $Y=A^{-\frac{1}{2}} B A^{-\frac{1}{2}}$ in the above inequality and multiplying both sides by $A^{\frac{1}{2}}$, we can get (4.8).

Substituting $A^{-1}$ for $A$ and $B^{-1}$ for $B$ in (4.8) and then taking the inverse of both sides, we get (4.9).

Theorem 4.4 Let $A, B \in \mathcal{B}^{++}(H)$ and $(1-v) A+v B \in \mathcal{B}^{++}(H)$ for $v \notin[0,1]$.

(I) If $s \in[0,1]$, then

$$
\begin{aligned}
& A \#_{s}\left(A \nabla_{\nu} B\right) \leq A \nabla_{\nu}\left(A \#_{s} B\right), \\
& A \#_{s}\left(A !_{\nu} B\right) \geq A !_{\nu}\left(A \#_{s} B\right) .
\end{aligned}
$$

(II) If $s \in[-1,0] \cup[1,2]$, then

$$
\begin{aligned}
& A \#_{s}\left(A \nabla_{\nu} B\right) \geq A \nabla_{\nu}\left(A \#_{s} B\right), \\
& A \#_{s}\left(A !_{\nu} B\right) \leq A !_{\nu}\left(A \#_{s} B\right) .
\end{aligned}
$$

Proof By the inequality (4.7) and using the same ideas as in the proof of Theorem 4.2, we can deduce this theorem. 
Competing interests

The authors declare that they have no competing interests.

\section{Authors' contributions}

All authors contributed equally to the writing of this paper. All authors read and approved the final manuscript.

\section{Acknowledgements}

The authors wish to express their heartfelt thanks to the referees for their detailed and helpful suggestions for revising the manuscript. The authors are grateful to Dr. Pujun Long for fruitful discussion and revising the manuscript.

Received: 30 March 2015 Accepted: 15 June 2015 Published online: 01 July 2015

\section{References}

1. Furuta, T, Mićić Hot, J, Pečarić, J, Seo, Y: Mond-Pečarić Method in Operator Inequalities. Element, Zagreb (2005)

2. Furuichi, S: On refined Young inequalities and reverse inequalities. J. Math. Inequal. 5, 21-31 (2011)

3. Kittaneh, F, Manasrah, Y: Reverse Young and Heinz inequalities for matrices. Linear Multilinear Algebra 59, 1031-1037 (2011)

4. Wu, J, Zhao, J: Operator inequalities involving improved Young and its reverse inequalities. J. Math. Anal. Appl. 421, 1779-1789 (2015)

5. Zuo, H, Shi, G, Fujii, M: Refined Young inequality with Kantorovich constant. J. Math. Inequal. 5(4), $551-556$ (2011)

6. Sagae, M, Tanabe, K: Upper and lower bounds for the arithmetic-geometric-harmonic means of positive definite matrices. Linear Multilinear Algebra 37, 279-282 (1994)

7. Mond, B, Pečarić, JE: A mixed arithmetic-mean-harmonic-mean matrix inequality. Linear Algebra Appl. 237/238, 449-454 (1996)

8. Newman, M: Kantorovich's inequality. J. Res. Natl. Bur. Stand. 64B, 33-34 (1960)

9. Mitroi, FC: About the precision in Jensen-Steffensen inequality. An. Univ. Craiova, Ser. Mat. Inform. 37(4), 73-84 (2010)

10. Bhatia, R: Matrix Analysis. Springer, New York (1997)

11. Krnić, M, Lovričević, N, Pečarić, J: Jensen's operator and applications to mean inequalities for operators in Hilbert space. Bull. Malays. Math. Soc. 35, 1-14 (2012)

12. Mitrinović, DS, Lacković, I: Hermite and convexity. Aequ. Math. 28, 229-232 (1985)

13. Moslehian, MS: Matrix Hermite-Hadamard type inequalities. Houst. J. Math. 39(1), 177-189 (2013)

14. Shi, S: Convex Analysis. Shanghai Science Technique Publisher, Shanghai (1990) (in Chinese)

\section{Submit your manuscript to a SpringerOpen ${ }^{\odot}$ journal and benefit from:}

$\rightarrow$ Convenient online submission

Rigorous peer review

- Immediate publication on acceptance

Open access: articles freely available online

- High visibility within the field

- Retaining the copyright to your article 